\title{
Application of mobile-technology for disease and treatment monitoring of malaria in the "Better Border Healthcare Programme"
}

Pongthep Meankaew ${ }^{1}$, Jaranit Kaewkungwal ${ }^{1,2^{*}}$, Amnat Khamsiriwatchara ${ }^{1}$, Podjadeach Khunthong ${ }^{1}$, Pratap Singhasivanon ${ }^{2}$, Wichai Satimai ${ }^{3}$

\begin{abstract}
Background: The main objective of this study was to assess the effectiveness of integrating the use of cell-phones into a routine malaria prevention and control programme, to improve the management of malaria cases among an under-served population in a border area. The module for disease and treatment monitoring of malaria (DTMM) consisted of case investigation and case follow-up for treatment compliance and patients' symptoms.

Methods: The module combining web-based and mobile technologies was developed as a proof of concept, in an attempt to replace the existing manual, paper-based activities that malaria staff used in treating and caring for malaria patients in the villages for which they were responsible. After a patient was detected and registered onto the system, case-investigation and treatment details were recorded into the malaria database. A follow-up schedule was generated, and the patient's status was updated when the malaria staff conducted their routine home visits, using mobile phones loaded with the follow-up application module. The module also generated text and graph messages for a summary of malaria cases and basic statistics, and automatically fed to predetermined malaria personnel for situation analysis. Following standard public-health practices, access to the patient database was strictly limited to authorized personnel in charge of patient case management.
\end{abstract}

Results: The DTMM module was developed and implemented at the trial site in late November 2008, and was fully functioning in 2009. The system captured 534 malaria patients in 2009. Compared to paper-based data in 2004-2008, the mobile-phone-based case follow-up rates by malaria staff improved significantly. The follow-up rates for both Thai and migrant patients were about 94-99\% on Day 7 (Plasmodium falciparum) and Day 14 (Plasmodium vivax) and maintained at 84-93\% on Day 90. Adherence to anti-malarial drug therapy, based on self-reporting, showed high completion rate for $P$. falciparum-infected cases, but lower rate for $P$. vivax cases. Patients' symptoms were captured onto the mobile phone during each follow-up visit, either during the home visit or at Malaria Clinic; most patients had headache, muscle pain, and fatigue, and some had fever within the first follow-up day (day $7 / 14$ ) after the first anti-malarial drug dose.

Conclusions: The module was successfully integrated and functioned as part of the malaria prevention and control programme. Despite the bias inherent in sensitizing malaria workers to perform active case follow-up using the mobile device, the study proved for its feasibility and the extent to which community healthcare personnel in the low resource settings could potentially utilize it efficiently to perform routine duties, even in remote areas. The DTMM has been modified and is currently functioning in seven provinces in a project supported by the WHO and the Bill \& Melinda Gates Foundation, to contain multi-drug resistant malaria on the Thai-Cambodian border.

\footnotetext{
* Correspondence: tmjkk@mahidol.ac.th

${ }^{1}$ Center of Excellence for Biomedical and Public Health Informatics (BIOPHICS), Faculty of Tropical Medicine, Mahidol University, Bangkok

Thailand

Full list of author information is available at the end of the article
} 


\section{Background}

The World Health Organization (WHO) has set ultimate goal to fight against malaria towards the elimination of the disease by which starts with good and effective malaria control programme [1,2]. Several control measures and interventions have been developed and implemented across the region, including mosquito control, indoor residual spraying, insecticide-treated nets, prompt and effective treatment, intermittent preventive prophylaxis, and behavioural change education $[3,4]$. One of the key strategies for eliminating malaria is the prompt identification and treatment of malaria patients. To achieve this goal, an effective diseasemanagement system should exist to enable rapid and accurate malaria case detection in target areas, and ensure effective treatment. Therefore, an effective system should allow case detection for early treatment at the point-of-care, and real-time case investigation and active follow-up of positive cases at the community level $[5,6]$. Although the vertical control programme administered by the Bureau of Vector-borne Diseases (BVBD) in Thailand is well organized and distributed throughout epidemic areas across the country, preventive and treatment control measures may not cover fully and efficiently in rural and remote communities. Difficulties in those communities might include limited service access, due to poverty and illegal immigration status, which inhibit villagers' treatment-seeking behaviours [7].

In the BVBD's vertical malaria-control programme [8], malaria-case treatment and care are managed down to the village level. In villages in epidemic areas, village malaria volunteers (VMVs) and/or village health volunteers (VHVs) work at the point-of-care, malaria posts (MPs), which collaborate with staff at the official malaria clinic $(\mathrm{MC})$. At the upper levels, treatment and care are monitored vertically by the Vector-borne Disease Unit (VBDU), Vector-borne Disease Center (VBDC), up to the regional Offices of Disease Prevention and Control (ODPC), and BVBD. In passive case detection, the local febrile patients visit at MC/MP or VBDU office for diagnosis. The VBDU staff, however, also conduct periodic active case detection in their responsible villages. The infected cases are registered and investigated in more detail. According to the standard malaria case management practices of Thailand's Ministry of Public Health (MOPH), medication and follow-up days are different, corresponding with the type of infection. The first-line drugs for malaria treatment adopted by the MOPH adhere to the national guidelines of Thailand and the WHO [9-13]. Typical uncomplicated Plasmodium falciparum cases are treated with three days of artesunate, plus mefloquine on day 2 and day3; with recommended follow-up at days 7 and 28, with additional days 60 and 90 [13-16]. For typical Plasmodium vivax malaria cases, patients are treated according to recommended WHO and Thai MOPH practices, with chloroquine for three days and a 14-day course of primaquine to prevent relapse; with recommended follow-up on days 14 , 28,60 , and 90 [14,17-19].

A major challenge for malaria-control programme is the assurance of prompt and effective treatment. It has been suggested in the literature that monitoring of prompt diagnosis and effective management of acute clinical episodes with anti-malarial drugs are crucial to reducing morbidity and mortality [20-22]. The WHO suggests that standard guidelines on duration of followup for safety monitoring are needed in different regions $[11,23]$. In Thailand, designated malaria staff routinely assess safety and tolerability, and monitor clinical failures, in accord with WHO guidelines. Strategies to promote patient adherence would improve drug performance and thereby might help to prevent the rapid emergence of drug resistance [24,25].

This study was part of the project Application of Smart Phone in "Better Border Healthcare Programme" (BBHP) that was awarded by the Microsoft Research in early 2008. The main objective of this two-year project was to develop technology-based healthcare solutions that would increase the accessibility and affordability of treatment and care services to the under-served communities [26]. The BBHP modules were developed in part to correspond with the United Nations' Millennium Development Goals (MDG), and BVBD malarial-control indicators. One MDG targeting healthcare services included combating malaria and other diseases, while the BVBD had set its mission for prevention and control of malaria to eventually eliminate it. The module for Disease and Treatment Monitoring of Malaria (DTMM), one part of the BBHP project, was thus developed in an attempt to find a solution using mobile technology to alter treatment-seeking behaviours and facilitate better treatment and care for malaria patients in lowresource settings. Mobile technology, particularly the cellular phone, has not only penetrated the daily lives of people in metropolitan areas and large rural cities/towns; they have also become popular among those living in remote areas. Thus the DTMM combined both web-based and smart-phone applications to manage malaria treatment and care activities, integrated into routine case detection and follow-up at selected MCs and VBDU. The main objective of this study was to assess the module's effectiveness in improving case follow-up and treatment compliance among an under-served population in a border area.

\section{Methods}

\section{Setting and study population}

The DTMM was implemented in pilot areas of Sai Yok District, western Kanchanaburi Province, along the 
Thai-Myanmar border. The district, with an area of $2,728.922 \mathrm{sq} \mathrm{km}(1,053.6 \mathrm{sq} \mathrm{mi})$, is divided into seven sub-districts, including 55 villages; there are 11 health centers, two sub-district hospitals, two VBDUs, and three MCs in the district $[27,28]$. The DTMM study areas covered three sub-districts (Bongti, Sri Monkol, and Lum-Sum), 13 main villages, plus some hamlets of other nearby villages, from which inhabitants sought treatment under the treatment coverage area of one $\mathrm{MC}$ and one VBDU. Most residents in the area are of Thai, Karen, Mon, and Myanmar ethnicity, and are farmers, woodcutters, and laborers. The non-Thai or migrants were classified as M1, or migrant workers who had been living or working in Thailand $\geq 6$ months; and M2, or migrant workers who had been living or working in Thailand $<6$ months.

At-risk groups for malaria transmission in the study area included Thais and migrants who resided along the border, and also uncontrolled and undocumented crossborder migrants. The latter group also sought treatment from the MP/MC in the area [29,30]. According to the BVBD stratification system [7], the types of transmission area are divided by control indicators; A1 (perennial malaria transmission area) is an area where transmission is reported $\geq 6$ months per year; A2 (periodic malaria transmission area) is an area where transmission is reported $\leq 5$ months per year; B1 (high-risk area) is where transmission has not been reported within the last three years, but primary and secondary vectors are found, and consequently, the area is potentially suitable for malaria transmission (high and moderate receptivity); B2 (low risk area) is where transmission has not been reported within the last three years; neither primary nor secondary vectors are found, and suspected vectors may be found (low and non-receptive).

Sai Yok District is located in a region with a tropical climate, typically with a rainy season in May-November, when most malaria cases are present; however, cases have been reported throughout the year in this malariatransmission zone. The three sub-districts where the DTMM was implemented, were classified as A1 areas. The annual parasite incidence (API) of malaria in Sai Yok District in 2009 was 17.3/1,000 population; Bongtee sub-district had the highest API, of $126.1 / 1,000$ population [31,32]. It was reported that $P$. falciparum and $P$. vivax were present in roughly equal proportions [31].

\section{DTMM module}

The DTMM was developed under the standard software development life cycle (SDLC) approach to cover malaria case management at the local community MCs and VBDU. This innovative module was designed to be consistent with the existing paper-based workflow to avoid resistance from changing the ways in which malaria staff performed their routine treatment and care activities. To make it more feasible for effective utilization in a field setting, some new features were added to the module while some redundant parts related to data collection were eliminated. The DTMM adopted mobile technology-based features to replace the standard manual paper-based methods of case detection and follow-up in the villages. Figure 1 shows the conceptual framework and workflow of the DTMM.

The three main functions of the DTMM are case detection/registry, new case investigation, and case follow-up. The case detection/registry and investigation functions of the DTMM have been adapted from the standard paper-based data collection of the infected patients. In the case-investigation form, details of case characteristics, type of malaria, and treatment, are collected. After a patient receives medication per standard treatment guidelines, a follow-up schedule is generated and updated each time follow-up is performed. Once the data have been entered into the module, each individual case, or list of registered or followed-up patients in the system, who have visited the MC/VBDU, can be examined by the responsible staff (see Figure 2).

Responsible malaria staff at the local treatment sites are provided with mobile phones loaded with the follow-up application module; they use the case-follow-up function of the DTMM to update the follow-up status on the schedule date, and capture the locations (coordinates) each time local malaria staff perform routine home visits. This replaces paper-based case-tracking in the villages, and supersedes routine map-drawing for case locations. The DTMM also sends home-visitschedule reminder messages directly to responsible local staff. During a home visit, the DTMM follow-up function can record the typical clinical symptoms of malaria. Information can be captured on the phone in areas without a telephone signal, and malaria-control staff can later synchronize information onto the system at the MC/VBDU, or wherever a signal is available (see Figure 3).

The use of the DTMM allows remote data transfer technology in both textual and geographic format. On a weekly basis, the system would generate short message service (SMS) with a summary of malaria cases, and automatically feed them to predetermined MC/VBDU personnel. A map of each scheduled visit can be displayed by clicking on the visit schedule table; this helps to locate cases in the area, and is especially useful for identifying foreign cases in remote border areas. Maps of all cases covered by the health service areas may be seen at the MC, and also at the upper supervisory level. Summary statistics can be generated to help malaria authorities make informed decisions and act accordingly (see Figure 4). 


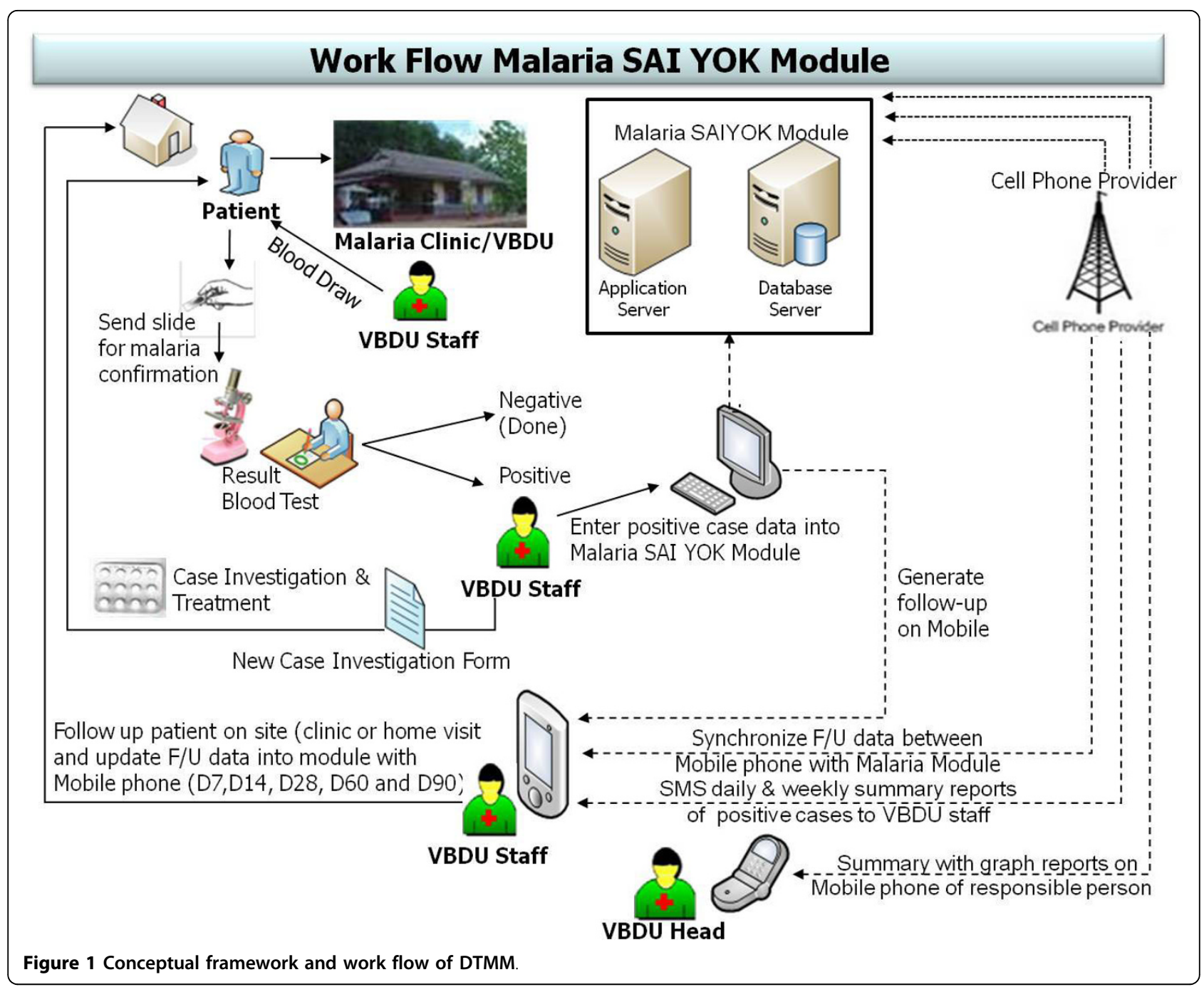

\section{Ethical considerations}

It should be noted that all patient-management activities and the database containing information associated with such activities, were strictly secured and used only by authorized personnel who were in charge of patient case-management. The electronic DTMM system maintains the same crucial data-integrity and confidentiality features as the paper-based processes at the local malaria clinics.

No written informed consent forms were signed by the patients who visited the malaria clinics, since the activities were all routine malaria clinic work; however, the staff had verbally informed the patients and asked them to return to the clinic, or agree to home visits, as part of their scheduled follow-up. Data extracted from the DTMM databases were secondary data with no identification linked to them. The authors requested official permission from the BVBD Director to use the extracted data for analysis. This study protocol was reviewed and approved by the Ethics Committee of the Faculty of Topical Medicine, Mahidol University. Electronic data were encrypted for transfer, to ensure confidentiality and security.

\section{Results}

\section{Malaria case investigation}

The DTMM module was developed and implemented at the trial site in late November 2008 and was fully functioning in 2009. During 2009, 534 malaria patients, 324 (61\%) Thais, and 210 (39\%) migrants, visited the two malaria clinics in Sai Yok area (Table 1). Almost all of the migrants were Karen and other minority groups who resided in Thailand for $\geq 6$ months (M1). The percentage of patients with P. falciparum (56\%) appeared to be slightly higher than $P$. vivax (44\%) among the Thais, while the percentages of $P$. falciparum and $P$. vivax were about the same among the migrants. Some cases had a history of infection, and used to visit the 


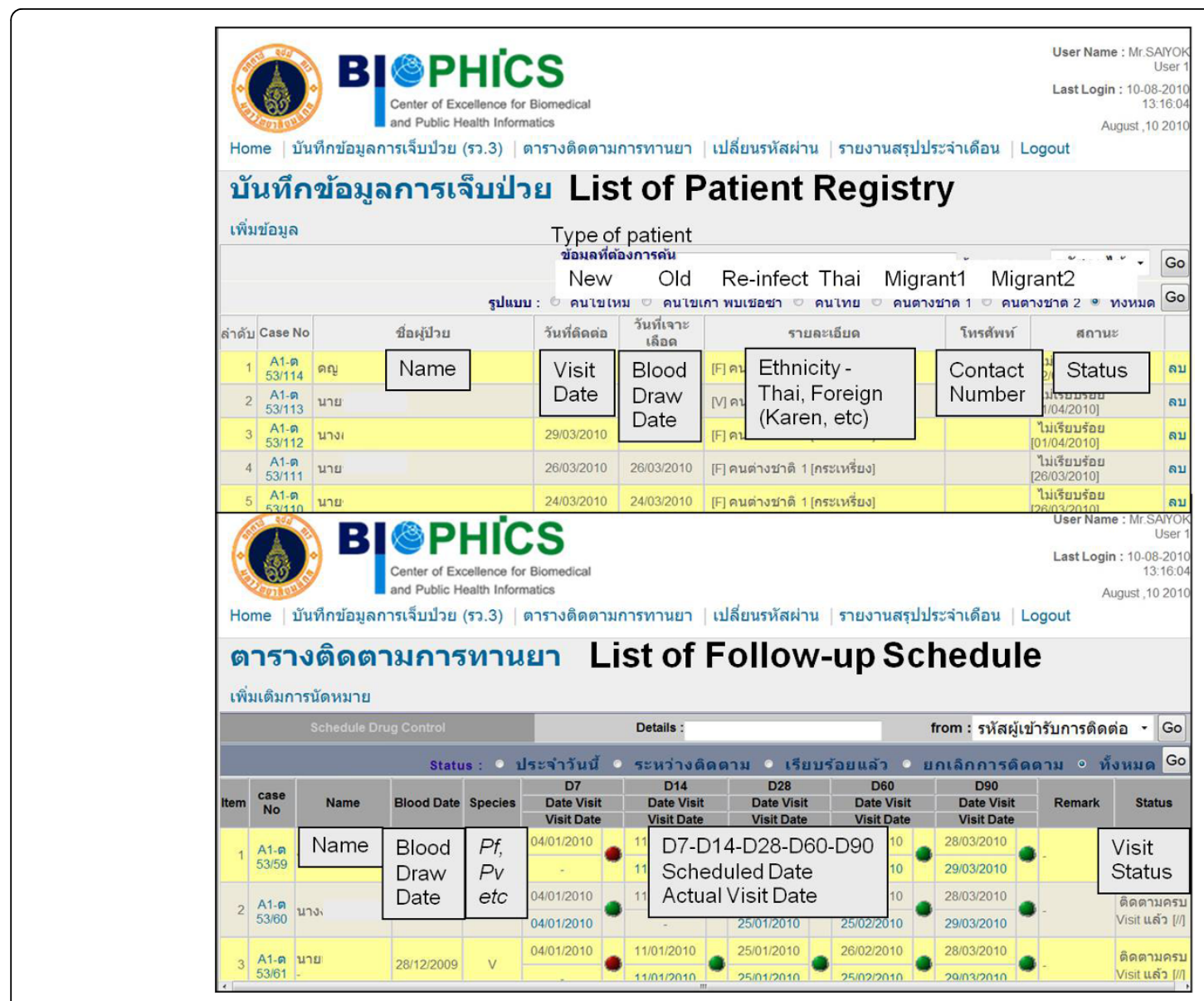

Figure 2 Screen shots of case registry and follow-up schedule.
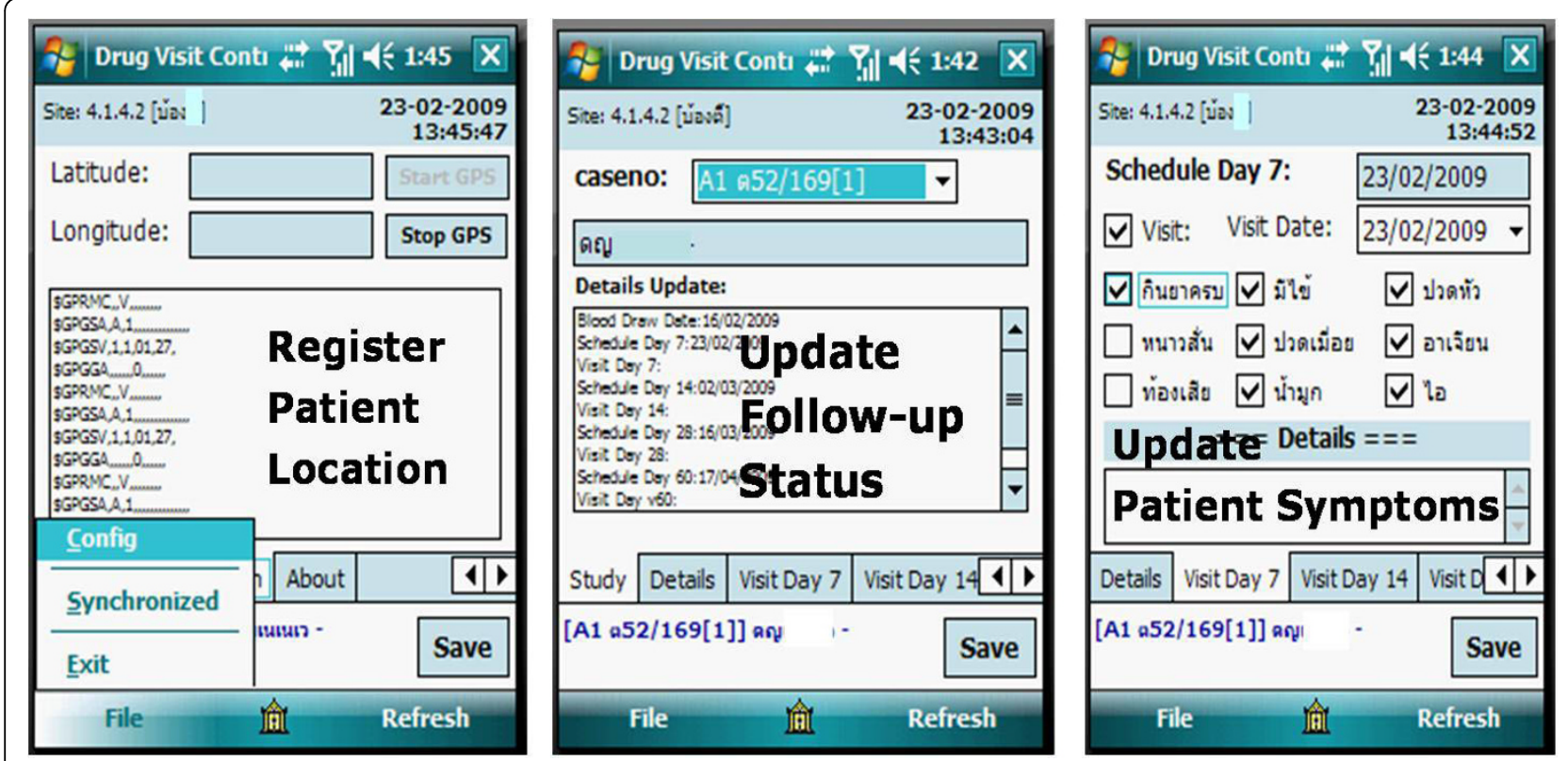

Figure 3 Screen shots of case follow-up on mobile phone 


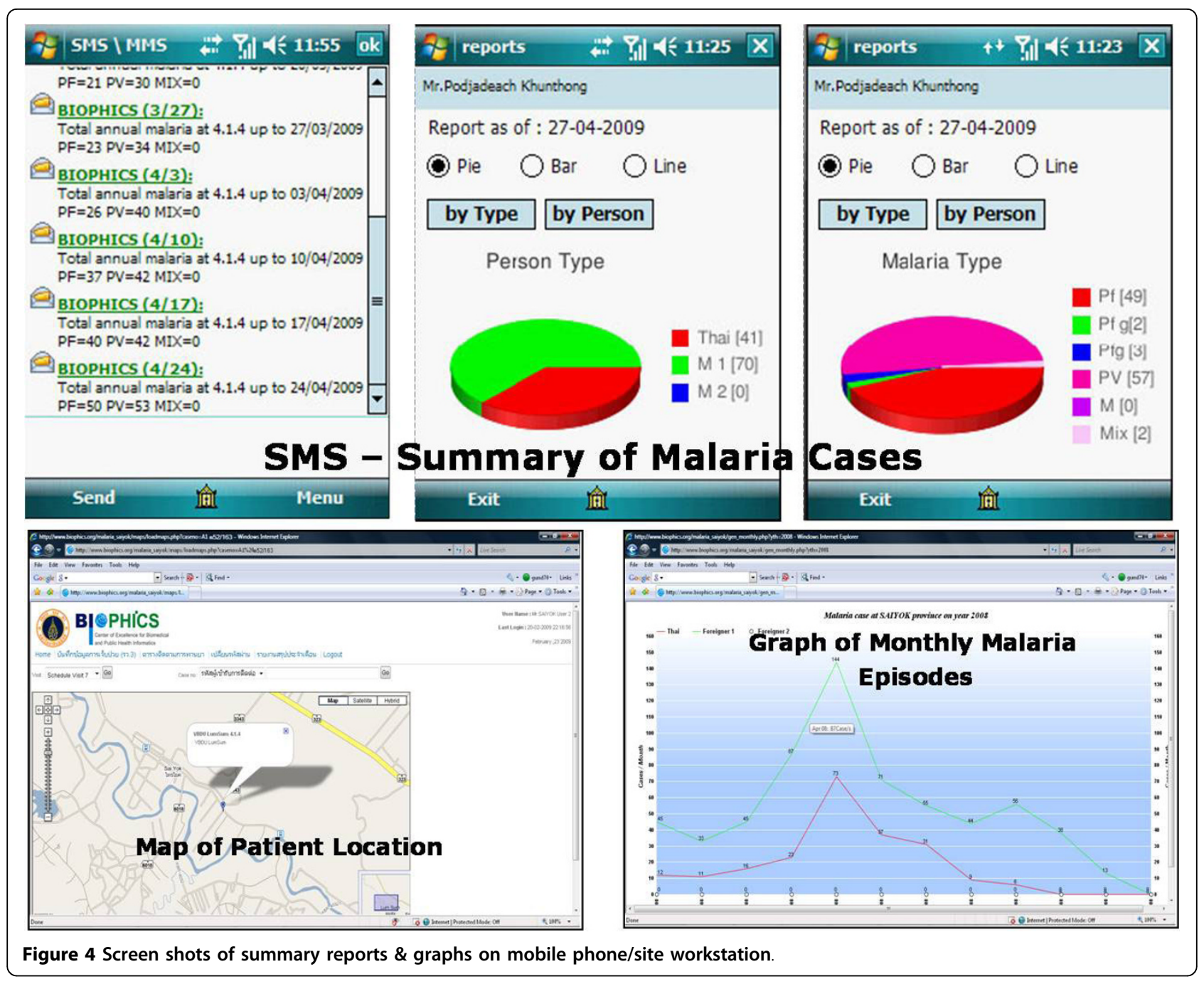

MCs (classified by the local malaria staff as "not cured" or "re-infected" cases); the percentage of old cases among the migrants (15.3\%) was higher than the Thais (7.1\%).

Over $70 \%$ of patients were male; most of them worked in rice and corn farms in the area. The average age of the Thai patients (Mean $22.1 \pm$ SD 15.4; Median 19, Range 83) was significantly different from the migrants (Mean $31.3 \pm$ SD 16.8; Median 30, Range 77). Among the Thais, more patients were children aged $<10$ years (28\%) than among the migrants (9\%). By contrast, among the migrants, more patients were elderly than among the Thais.

Case investigation by malaria staff, attempting to explore possible risk factors based on self-reporting, found that $41 \%$ of migrants and $34 \%$ of Thais did not leave their households or current places of residence before experiencing febrile symptoms; these could be indigenous cases. Interestingly, self-reports of leaving the household 1-2 days before presumable infection, showed that more migrant patients stayed elsewhere in the province (47\%) than Thais (29\%), while more Thais (36\%) traveled across the border than migrants (11\%).

Almost everyone owned a bed-net to protect themselves from mosquito bite; over half of the families had $>1$ bed-net. About 70\% or more of both Thai and migrant patients reported that they usually slept in a bed-net when staying in a transmission area. About 50\% of both groups reported that they stayed in a house that had been sprayed.

\section{Malaria case follow-up}

Baseline data were collected for all malaria cases in the study area, for the years 2004-2008, before implementation of the DTMM (Table 2). The numbers of both Thai and migrant malaria patients varied; there appeared to be more migrant than Thai cases most years, and an outbreak was observed among migrants in 
Table 1 Case investigation of malaria patients in Sai Yok District, 2009

\begin{tabular}{|c|c|c|c|c|}
\hline \multirow[t]{2}{*}{ Characteristics } & \multicolumn{2}{|c|}{ Migrant $(n=210)$} & \multicolumn{2}{|c|}{ Thai $(n=324)$} \\
\hline & $n$ & $\%$ & $n$ & $\%$ \\
\hline \multicolumn{5}{|l|}{ Type of Infection } \\
\hline \multicolumn{5}{|l|}{ Species } \\
\hline Plasmodium falciparum & 104 & 49.5 & 181 & 55.9 \\
\hline Plasmodium vivax & 106 & 50.5 & 143 & 44.1 \\
\hline \multicolumn{5}{|l|}{ Type of patient } \\
\hline New case & 178 & 84.8 & 301 & 92.9 \\
\hline Old case - not cured & 6 & 2.9 & 4 & 1.2 \\
\hline Old case - reinfection & 26 & 12.4 & 19 & 5.9 \\
\hline \multicolumn{5}{|l|}{ Personal characteristics } \\
\hline \multicolumn{5}{|l|}{ Gender } \\
\hline Male & 151 & 71.9 & 235 & 72.5 \\
\hline Female & 59 & 28.1 & 89 & 27.5 \\
\hline \multicolumn{5}{|l|}{ Age Group (Year) } \\
\hline$<=10$ & 19 & 9.0 & 91 & 28.1 \\
\hline $11-20$ & 57 & 27.1 & 89 & 27.5 \\
\hline $21-30$ & 30 & 14.3 & 59 & 18.2 \\
\hline $31-40$ & 35 & 16.7 & 45 & 13.9 \\
\hline $41-50$ & 36 & 17.1 & 19 & 5.9 \\
\hline $51-60$ & 22 & 10.5 & 17 & 5.2 \\
\hline$>=61$ & 11 & 5.2 & 4 & 1.2 \\
\hline \multicolumn{5}{|l|}{ Occupation } \\
\hline Pre-school child & 10 & 4.8 & 35 & 10.8 \\
\hline Student & 32 & 15.2 & 90 & 27.8 \\
\hline Agricultural - farming & 131 & 62.4 & 172 & 53.1 \\
\hline Work in forest - cut wood, hunting & 22 & 10.5 & 17 & 5.2 \\
\hline Commercial & 8 & 3.8 & 3 & 0.9 \\
\hline Labor & 4 & 1.9 & 7 & 2.2 \\
\hline Other & 3 & 1.4 & 0 & 0.0 \\
\hline \multicolumn{5}{|l|}{ Marital status } \\
\hline Not married & 90 & 42.9 & 207 & 63.9 \\
\hline Married & 120 & 57.1 & 117 & 35.1 \\
\hline \multicolumn{5}{|l|}{ Risk factors } \\
\hline \multicolumn{5}{|l|}{ Stayed out of household (before infection) } \\
\hline No & 87 & 41.4 & 111 & 34.3 \\
\hline Yes - within town/province & 99 & 47.1 & 95 & 29.3 \\
\hline Yes - in Myanmar & 24 & 11.4 & 116 & 35.8 \\
\hline Yes - not specified & 0 & 0.0 & 2 & 0.6 \\
\hline \multicolumn{5}{|l|}{ Own bed-net } \\
\hline Not own any bed-net & 7 & 3.3 & 7 & 2.2 \\
\hline Own at least 1 bed-net & 203 & 96.7 & 317 & 97.8 \\
\hline \multicolumn{5}{|l|}{ Amount of bed-nets in household } \\
\hline 1 & 47 & 22.4 & 142 & 43.8 \\
\hline $2-3$ & 147 & 70.0 & 165 & 51.0 \\
\hline $4-5$ & 7 & 3.4 & 10 & 3.0 \\
\hline \multicolumn{5}{|c|}{ Sleep in bed-net when staying in transmission area } \\
\hline No & 64 & 30.5 & 75 & 23.1 \\
\hline Yes & 143 & 68.1 & 246 & 75.9 \\
\hline Not specified & 3 & 1,4 & 3 & 0.9 \\
\hline \multicolumn{5}{|l|}{ Staying in sprayed household } \\
\hline No & 98 & 46.7 & 154 & 47.5 \\
\hline Yes & 109 & 51.9 & 168 & 51.9 \\
\hline Not specified & 3 & 1.4 & 2 & 0.6 \\
\hline
\end{tabular}


Table 2 Malaria case follow-up, 2004-2009

\begin{tabular}{|c|c|c|c|c|c|c|}
\hline \multirow[t]{3}{*}{ Year } & \multicolumn{3}{|c|}{ P. falciparum } & \multicolumn{3}{|c|}{ P. vivax } \\
\hline & \multirow[t]{2}{*}{ Total cases } & \multicolumn{2}{|c|}{$\%$ Follow-up } & \multirow[t]{2}{*}{ Total cases } & \multicolumn{2}{|c|}{$\%$ Follow-up } \\
\hline & & Day 7 & Day 28 & & Day 14 & Day 28 \\
\hline \multicolumn{7}{|l|}{ Thai } \\
\hline 2009 & 181 & 99.4 & 98.3 & 143 & 97.9 & 97.9 \\
\hline 2008 & 161 & 36.7 & 26.1 & 110 & 33.6 & 26.4 \\
\hline 2007 & 115 & 32.2 & 20.9 & 99 & 35.4 & 21.2 \\
\hline 2006 & 133 & 38.4 & 22.6 & 104 & 24.0 & 12.5 \\
\hline 2005 & 113 & 27.4 & 22.1 & 81 & 32.1 & 19.8 \\
\hline 2004 & 209 & 36.4 & 30.1 & 186 & 37.1 & 37.1 \\
\hline \multicolumn{7}{|l|}{ Migrant } \\
\hline 2009 & 104 & 93.9 & 97.0 & 106 & 98.1 & 98.1 \\
\hline 2008 & 444 & 25.2 & 5.6 & 231 & 25.1 & 6.1 \\
\hline 2007 & 293 & 11.6 & 12.9 & 101 & 5.1 & 8.9 \\
\hline 2006 & 312 & 17.9 & 9.3 & 106 & 27.4 & 13.2 \\
\hline 2005 & 227 & 19.8 & 18.5 & 77 & 31.2 & 20.8 \\
\hline 2004 & 205 & 30.7 & 32.7 & 167 & 35.3 & 28.7 \\
\hline
\end{tabular}

2008. Case follow-up percentages on Day 7 (for P. falciparum), Day 14 (for $P$. vivax), and Day 28 (both) during the period 2004-2008 also varied in the range $20-40 \%$ among the Thai cases, but could be $<10 \%$ among the migrants. The follow-up rates on Day 28 were usually lower than Day 7.

In 2009, after the DTMM had been implemented and was fully functioning, there were 534 malaria patients, 285 (53.3\%) P. falciparum, and 249 (46.7\%) P. vivax.
Compared with the baseline data, the follow-up rates collected by malaria staff on their mobile phones showed significant improvement. The rates for Thai $P$. falciparum and $P$. vivax cases were $>90 \%$ (range 98 99\%) at first follow-up day (Day 7 or Day 14), to 93\% at the last recommended follow-up day (Day 90) (see Figure 5). The rates of follow-up among the migrant patients were lower: $94 \%$ on Day 7 to $84 \%$ on Day 90 for P. falciparum, and $98 \%$ on Day 14 to $85 \%$ on Day 90 for $P$. vivax.

Self-reported adherence to anti-malarial drug therapy showed high completion rates for $P$. falciparum (94.0\%) but fairly low rates for P. vivax (42.6\%) (see Table 3). Patients' symptoms were captured onto the mobile phones during follow-up visits, either during home visits or at the MC/MP. At 7 days after the first anti-malarial drug dose, a high proportion $(83 \%)$ of $P$. falciparum patients reported headache, over half $(60 \%)$ reported muscle pain and fatigue, some (15\%) had fever, and about $17 \%$ vomited. Similarly at 14 days after the first anti-malarial drug dose, most $P$. vivax patients (72\%) reported headache, about half (51\%) reported muscle pain and fatigue, and some (12\%) had fever (Table 3).

The data in the DTMM could also be mapped, using a geographical information system (GIS). The geographical locations of cases captured on Day 7 (P. falciparum), Day 14 (P. vivax) and Day 28 (P. falciparum + P. vivax) are shown in Figure 6. No spatial or temporal analysis,

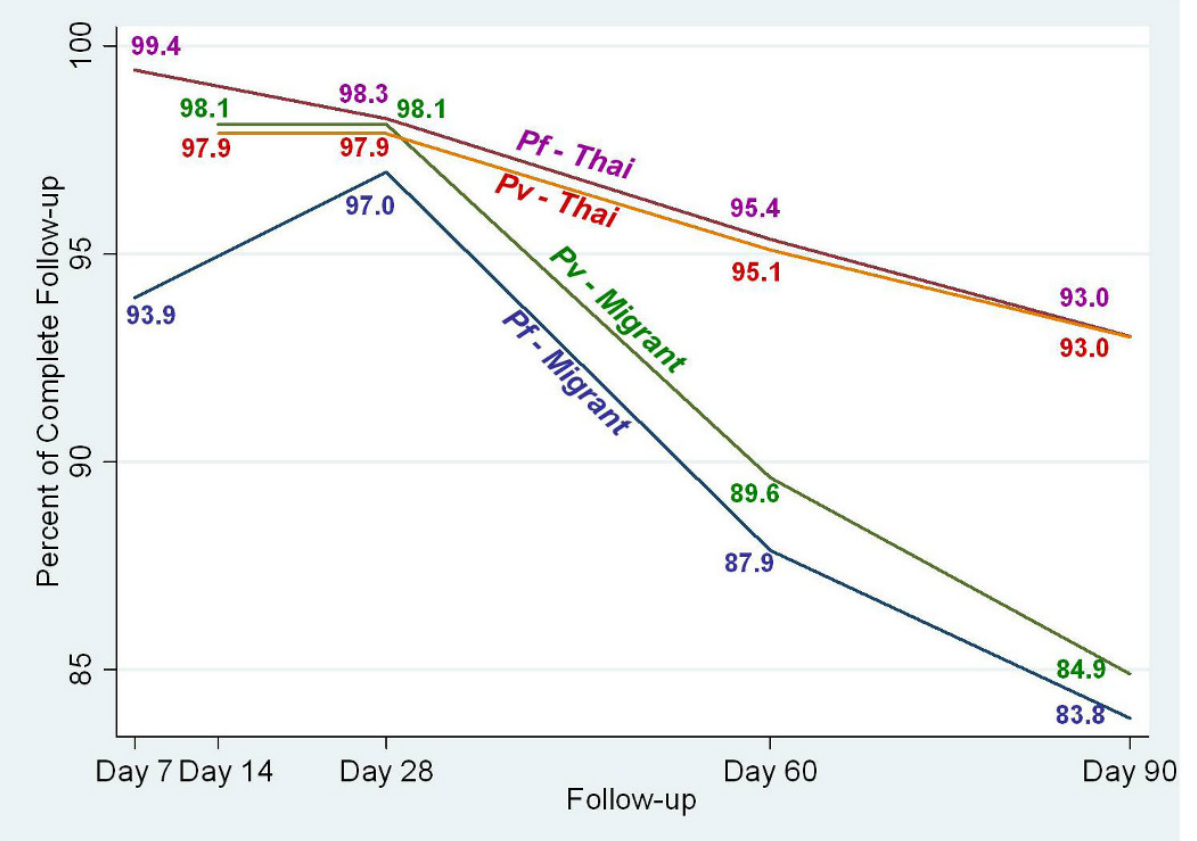

Figure 5 Complete follow-up rates of $P$. falciparum and $P$. vivax patients. 
Table 3 Malaria case follow-up for $P$. falciparum and P. vivax patients, Sai Yok District, 2009

\begin{tabular}{|c|c|c|c|c|}
\hline \multirow[t]{2}{*}{ Follow-up On Mobile Phone } & \multicolumn{2}{|c|}{ P. falciparum cases $(n=285)$} & \multicolumn{2}{|c|}{$P$. vivax cases $(n=249)$} \\
\hline & $\mathbf{n}$ & $\%$ & $\mathbf{n}$ & $\%$ \\
\hline \multicolumn{5}{|l|}{ Complete treatment (self-reported) } \\
\hline No & 17 & 6.0 & 143 & 57.4 \\
\hline Yes & 268 & 94.0 & 106 & 42.6 \\
\hline \multicolumn{5}{|c|}{ Symptoms within the first follow-up day (day $7 /$ day 14 ) post first dose } \\
\hline Fever & 42 & 14.7 & 29 & 11.6 \\
\hline Headache & 235 & 82.5 & 179 & 71.9 \\
\hline Chill & 10 & 3.5 & 6 & 2.4 \\
\hline Muscle Pain/Fatigue & 172 & 60.4 & 127 & 51.0 \\
\hline Vomit & 49 & 17.2 & 16 & 6.4 \\
\hline Diarrhea & 5 & 1.8 & 2 & 0.8 \\
\hline
\end{tabular}

as part of the initial version of DTMM, was used in this study. However, the simple disease mapping of these cases at the village location could help malaria staff target preventive action more accurately within the identified high-risk zones. Mapping malaria-risk locations makes it possible to conduct active case management, allocate priorities in control programmes, and plan interventions.

\section{Discussion}

\section{Malaria cases among border populations}

In 2009, in contrast with the previous 5 years, the numbers of malaria cases among Thais in the study areas were higher than among migrant cases who had mostly resided in Thailand $\geq 6$ months. The very high numbers of migrant patients in 2008, pre-DTMM, was due to the high cross-border population numbers that particular year. Baseline information during the 5 preDTMM years usually showed more $P$. falciparum than $P$. vivax cases; but for the year 2009 , the proportion of $P$. falciparum-infected cases was higher among Thais but similar among migrants. Meanwhile, a history of malaria infection was lower among Thais than migrants. These proportions of infection captured from the DTMM reflected disease burden in the areas even though it derived only from symptomatic individuals who came to malaria clinics seeking anti-malarial treatment. Almost all cases in border areas would seek treatment at governmental malaria clinics or the district hospital, since there was no private hospital in the area, and anti-malarial drugs are not available in Thai drug stores. In an epidemiological study conducted in a low-transmission setting on the Thai-Myanmar border [33], it was reported that all P. falciparum infections were symptomatic, but approximately $10 \%$ of $P$. vivax infections were still asymptomatic. In another cross-sectional study in intense malaria transmission districts near the site of this study, along the ThaiMyanmar border [34], among the surveyed 125 households, $10 \%$ were Thai, $42 \%$ Mon, and $48 \%$ Karen; about $40 \%$ of Thai and Karen migrants and almost $30 \%$ of Mon migrants reported having suffered from malaria at least once.

The age and sex distribution of the malaria patients reflected the disease situation in the area. Most patients in both groups were working-aged males who laboured in rice and corn farms in the area, while some reported working in forest areas along the border. In a retrospective study along Thailand's borders [35], increasing proportions of malaria cases were shown over 12 years among cross-border migratory foreign workers; these cases were especially concentrated in districts bordering Cambodia and Myanmar. This trend suggested that cross-border seasonal labour might play an important role in malaria transmission. In the other study of factors influencing self-reported malaria among migrants living along the Thai-Myanmar border [34], migrant working conditions played a major role in acquiring malaria.

In contrast with their infection status, most Thai and migrant patients in this study reported that they owned a bed-net and usually slept in a bed-net when they stayed in a transmission area. In a study following 3 years' bed-net use by villagers in Kanchanaburi Province [36], it was reported that conventional insecticidetreated mosquito nets (ITN) had been used over the years by the villagers, but that they needed to be retreated promptly at 6-month intervals, or they would only provide $15 \%$ protection against malaria vectors. The results of the current study may reflect the findings of the bed-net study such that even though patients claimed to use bed-nets when they stayed in a transmission area, the ITNs might fail due to being re-treated improperly. A long-term follow-up study on bed-net use [36] also reported that ITN implementation at community level had several technical, operational, economic, and social difficulties, and as a result, low re-treatment rates were observed. 


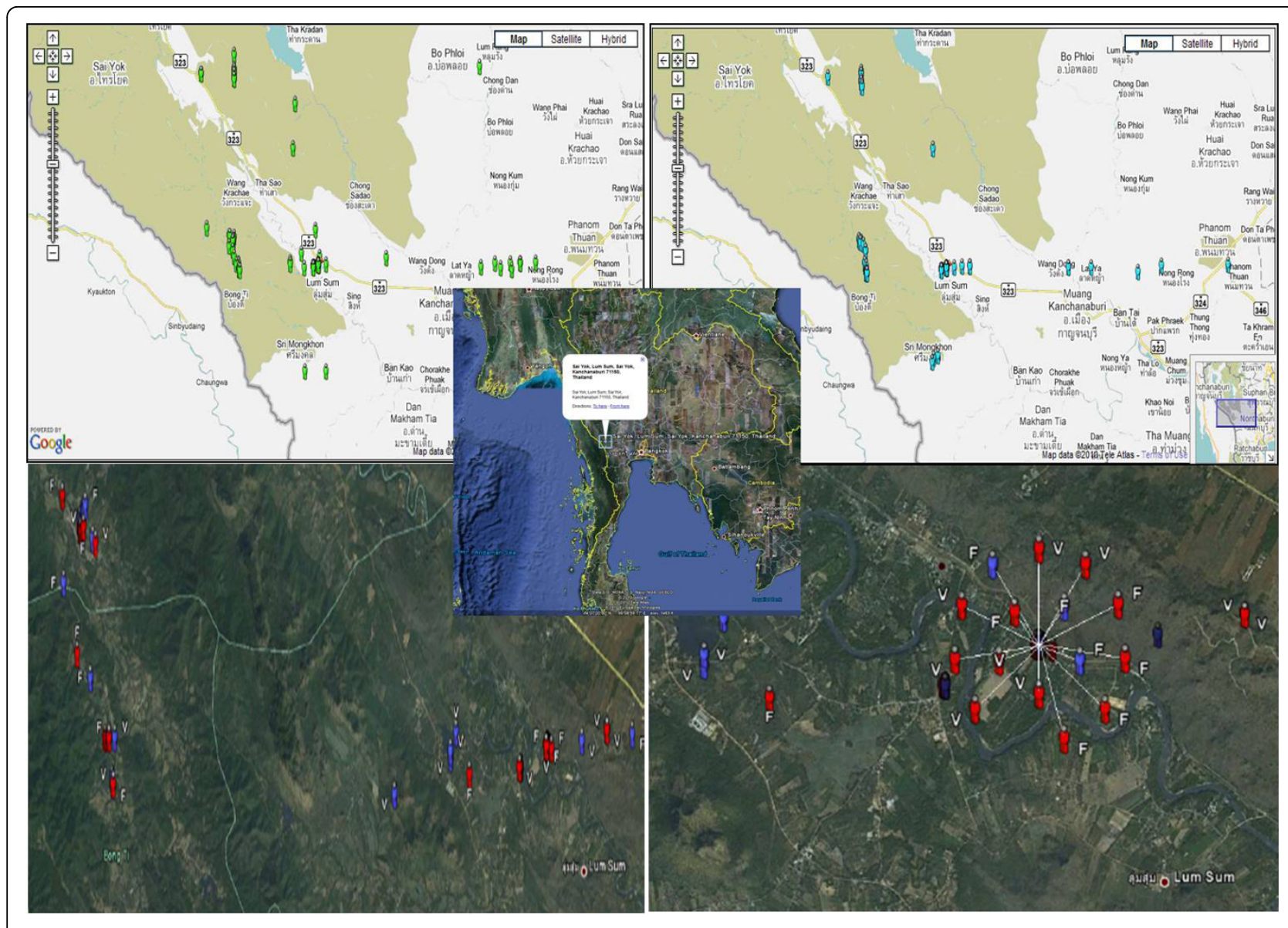

Figure 6 Screen shots of disease mapping of follow-up cases.

\section{Adherence to treatment and care}

For self-reported adherence to anti-malarial drug therapy, this study found a completion rate of $>90 \%$ for $P$. falciparum cases. This markedly rate may due to the short course of treatment; however, this figure was based on self-reports, not direct observations, and may not be completely reliable. In contrast, adherence to treatment among the $P$. vivax cases was $<50 \%$, probably due to the longer treatment course. Similarly, a study of 206 P. vivax patients on the northern Thai-Myanmar border [37] found $76 \%$ did not complete the full medication course. A study on adherence to treatment among $P$. vivax patients found significant differences in knowledge about malaria and perceptions of the benefits of anti-malarial drugs between adherent and nonadherent groups.

Like any public health programme, the success or failure of a malaria control programme is largely determined by the effective use of the services offered to the public. Monitoring the prompt use of effective drugs would greatly reduce the incidence of severe and complicated disease [22]. Effective, active case monitoring can also increase the chances of capturing relapse and reinfection. In this study, the use of mobile phones, as part of the DTMM, proved effective in increasing case follow-up rates, in line with the days recommended for ideal case-management and monitoring (up to day 90). The follow-up rates for P. falciparum and $P$. vivax Thai patients were almost $100 \%$ at first follow-up day (Day $7 /$ 14 ), and were maintained at $>90 \%$ to Day 90 . Even though the follow-up rates for migrant patients appeared lower, at about $85 \%$ at Day 90 , this could still be considered a marked success. Case follow-up rates using the smartphone in the DTMM were much higher than the five pre-DTMM years. The increases in followup rates for migrant workers has made it a promising tool for planning malaria prevention and control programmes in the cross-border multi-drug-resistant malaria containment project.

However, it is possible that the treatment adherence and case-follow-up rates observed in this study may be overestimated. Although the DTMM activities attempted to mimic the routine malaria prevention and control programme situation, efforts to conduct home visits by 
malaria staff may have been more diligent than normal practices in case management due to sensitizing with the programme and equipment. Nevertheless, this finding reflects what malaria case management could have achieved in terms of promoting adherence to therapy and case monitoring in ideal situation and beyond what will generally be expected for case follow-up to Day 28 .

\section{Monitoring signs and symptoms}

During routine follow-up home visits, non-specific symptoms and signs of malaria (fever, headache, shivering/chills, muscle pain/fatigue, diarrhea, and vomiting) were captured on mobile phone. The purpose of this DTMM feature is to monitor the typical symptom of malaria which could be cyclical occurrence and to avoid chance of complicated incident as delay of diagnostic and treatment may lead to severe complications [38]. Other side-effects and severe adverse events (if any) were monitored by malaria staff outside the mobile tool. The information captured by the DTMM smartphone revealed that patient symptoms from first drug dose to first follow-up day were similar to those reported in several other clinical-trial studies [31,39,40].

These symptoms could also have effect on adherence to treatment previously discussed. Drug failure might occur in malaria endemic area by several means including, in part, the patients got relief from major clinical symptoms and thus not adhered to the full course. This could easily go undetected without an effective followup system. Propagation of resistant parasites could result in an epidemic without effective case monitoring and the lack of an early warning module [41].

\section{Conclusions}

\section{DTMM usage}

The DTMM has shown successfully integrated into the malaria control programme's operations at the pilot sites. Data in the secured database of the module helped local malaria staff at the MC conduct their activities. The DTMM has succeeded in providing information to local malaria staff for routine case-management activities, and in providing timely summary reports for situational analysis. The DTMM made data collection easier at the local clinic, and additional data were collected on the mobile phone during routine home visits. With more information collected systematically in the database, this module has allowed the exploration of clinical outcomes and the generation of new hypotheses about transmission, and prevention and control measures. It would also be a useful tool for intervention studies, since it can collect reliable and timely variables, and identify cases in study areas. It has the potential to help determine vulnerable predictors of malaria outbreaks.
In summary, malaria case-management using the DTMM appeared to have reached close to the target of national guideline, especially for case-monitoring and follow-up. By using information from the electronic-based data capture and data management with an additional feature of mobile technology for data transfer, follow-up could even be successfully performed beyond day 28 . The DTMM has made it easier and faster to collect and process data closer to real-time, compared with the original paper-based process. The module removes repetitive data collection and generates useful reports for malaria control actions. Malaria staff at the pilot site, who used the DTMM, expressed satisfaction with the module's applications. It should be noted, however, that it required several meetings and training sessions to familiarize staff with the DTMM, and to use the smartphone for data collection in areas with and without mobile telephone service. Another issue requiring attention is linkage of the DTMM to the existing healthcare system, making it part of Thailand MOPH's routine work practices. This could be achieved by making the local staff feel ownership and make use of the data collected locally while be able to generate the routine reports needed by $\mathrm{MOPH}$.

\section{Module operation and management costs}

In quantifying the costs of designing, implementing, and maintaining this module, certain factors must be taken into account. The estimates here comprise hardware and software costs, not personnel costs.

The DTMM was developed by BIOPHICS with support from a Microsoft Research Award; its concept and programming parts were posted as open-source. The DTMM requires a server to manage data processing (scheduling follow-up appointments and updating visits) and text message delivery. Currently, the server is located at BIOPHICS, but this can be put as part of the existing computer at VBDU or upper levels. In 2009 figures, the investment cost for the DTMM (minimum for hardware requirements, including server, workstation, and tape back-up) is about US $\$ 2,500$, and software licenses for Windows and SQL about US \$2,500. MC/ VBDU staff enter data into the DTMM system using a laptop (or workstation) connected to the internet by an Aircard (maintenance cost about US \$20 per month) with unlimited data package. The SMS cost was about US $\$ 0.03$ per message. At least one low-cost smartphone should be allocated to VBDU staff per operational area; the prices of smartphones have dropped dramatically over the year. In 2009, the DTMM smartphone cost approximately US $\$ 300$.

\section{Challenges}

The results of this study, and epidemiological data from other studies, show that the prevalence of malaria 
remains unacceptably high in border areas. The crucial groups requiring attention include hard-to-reach populations, ethnic minorities, and mobile populations. Surveillance data over the years imply that the provinces with the highest incidence rate border Myanmar and Cambodia. Population movements in these areas, together with high drug pressure, are considered responsible for the development and spread of drug-resistant $P$. falciparum malaria [35]. It has been suggested that efforts to isolate tolerant parasites and eliminate malaria require more harmonized and aligned multi-country malaria control and pre-elimination strategies [42].

Thus, in 2008, the WHO and the BVBD of Thailand $\mathrm{MOPH}$ developed a programme to contain multi-drug resistant malaria along the Thai-Cambodian border. In this work, recommendations were made to improve malaria treatment adherence by Directly Observed Treatment (DOT), to implement cross-border strategies to harmonize malaria-control strategies, and to share information on drug resistance and follow-up on patients who regularly crossed the border $[5,42]$. One strategy included an effective surveillance system, which could capture and manage malaria cases in border areas, by incorporating such vulnerable populations $[5,6,42]$. Therefore, the DTMM was modified and enhanced to be Malaria Information System (MIS) with the purpose to expand for implementation in seven provinces along the Thai-Cambodian border. The modified DTMM (the MIS) is now functioning particularly in response to emergency containment and in planning the elimination of tolerant malaria parasites in the region. The MIS is a collaborative effort between BIOPHICS and the BVBD of Thailand's $\mathrm{MOPH}$, and is funded by WHO and the Bill and Melinda Gates Foundation (BMGF) for 2009-2010 [43].

The MIS, the modified version of the DTMM, is functioning as a surveillance and case management system for malaria incidents at the local level. Like any surveillance system, its value for infectious disease is measured by its ability to provide timely, accurate "data for action" to people responsible for effective prevention and control activities, and by its ability to provide ongoing feedback to the primary gatherers of information [44]. It is believed that long-term and accurate datasets, such as that provided by the MIS, are essential for prediction and trend analysis of malaria. In the containment project, the web-based and mobile phone systems have been modified to capture clinical, laboratory, and parasitic outcomes. The enhanced version of the DTMM utilizes more GIS features and spatial analysis which are essential to focus on scarce resources, improve the efficacy of control, and decrease the burden of disease [45]. The modified DTMM also adds spatio-temporal presentations for decision-making and containment purposes. Specifically, within the modified DTMM system launched in the border area, GIS could be useful at both macro and micro levels in planning the provision of health infrastructure, mapping disease distribution, investigating the spatial dynamics of disease transmission, and modeling health service utilization and disease control intervention.

The major challenge for success of the system is data quality, including data integrity, completeness, and timeliness. Since the system is designed to capture data, in part, for migrants and hard-to-reach populations, it should be acknowledged that some malaria infections may not be contracted at the place of reporting. The MIS is in the process of enhancement, to enable it to handle such difficult situations. Dedicated staff were a crucial factor for success of the DTMM; at minimum, they should be well-trained in geographical-information and mobile-technology applications; while this is not difficult, it cannot be over-emphasized. This is an important factor to consider for MIS implementation in new border areas.

The MIS has now been functioning to the eastern Thai-Cambodia border, despite its origins on the western Thai-Myanmar border. Another challenge is to expand the modified version of the DTMM to manage a wider range of endemic areas back on the longer stretching western border. Strengthening MC and VBDU infrastructure and additional financial support are needed in certain locations for sustainability and implementation at a national level. It is a substantial challenge to expand and maintain such a system nationwide and to eventually be part of and managed by the Thailand MOPH malaria surveillance team. Moreover, the system design will need adjustment, so that it is more modular approach, and more easily adapted for use beyond Thailand's borders.

\section{Acknowledgements}

We would like to acknowledge the Sai-Yok District Vector-borne Disease Unit, Kanchanaburi Province, for their vision and collaboration in implementing the module; Dr Supawadee Poungsombat for sharing her experience with malaria surveillance systems and initial introduction to the pilot site; all local malaria personnel at malaria clinics in the pilot study areas for their devotion to providing treatment and care for malaria patients; the IT and data management teams at BIOPHICS for their contribution to system development and implementation, and Microsoft Research for their funding and excellent support throughout the project.

\section{Author details}

'Center of Excellence for Biomedical and Public Health Informatics (BIOPHICS), Faculty of Tropical Medicine, Mahidol University, Bangkok, Thailand. ${ }^{2}$ Department of Tropical Hygiene, Faculty of Tropical Medicine, Mahidol University, Bangkok, Thailand. 'Bureau of Vector-borne Diseases, Department of Disease Control, Ministry of Public Health, Nonthaburi, Thailand.

\section{Authors' contributions}

$\mathrm{PM}, \mathrm{AK}, \mathrm{PS}, \mathrm{JK}$ were involved in the conceptualization and design of the study, and design of the application module. PK designed and programmed the application module, monitored and maintained the module's 
implementation, and extracted data for analysis. WS was responsible for managing and supervising the overall malaria-control-programme activities. AK and PM were in charge of monitoring progress of the module application. PM and JK performed statistical analyses and drafted the manuscript. All authors read and approved the final manuscript.

\section{Competing interests}

The authors declare that they have no competing interests. Development of the DTMM was supported by Microsoft Research.

Received: 22 June 2010 Accepted: 19 August 2010

Published: 19 August 2010

\section{References}

1. World Health Organization: Malaria elimination: A field manual for low and moderate endemic countries. Geneva: WHO 2008, ISBN 978924 1596084.

2. World Health Organization: Global malaria control and elimination: report of a technical review. Geneva: WHO 2008, ISBN 9789241596756.

3. World Health Organization: Regional action plan for malaria control and elimination in the western pacific (2010-2015). Geneva: WHO 2009.

4. Lines J, Whitty CJM, Hanson K: Prospects for eradication and elimination of malaria: A technical briefing for DFID, December 2007. British Government's Department for International Development (DFID) 2007.

5. World Health Organization: Containment of malaria multi-drug resistance on the Cambodia-Thailand border. Report of an Informal Consultation Phnom Penh 2007

6. World Health Organization: Global malaria report. Geneva: WHO 2008 [http://www.malaria.who.int/wmr2008].

7. Thailand Country Coordination Mechanism: Partnership towards malaria reduction in migrants and conflict-affected populations in Thailand. CCM-Thailand Round 9 malaria proposal to the GFATM 2009.

8. World Health Organization: Consultative Meeting on Human Resource on Vector Borne Disease. SEAMEO TROPMED Network Office, Bangkok Thailand 2008.

9. Sittichai N: Antimalarial drugs: A current situation. Thai J Pharm Sci 2004, 28(3-4):95-110.

10. World Health Organization: Guidelines for the treatment of malaria. Geneva: WHO 2006 [http://whqlibdoc.who.int/publications/2006/ 9241546948_eng.pdf], Document No. WHO/HTM/MAL.2006.1108.

11. World Health Organization: Guidelines for the treatment of malaria. Geneva: WHO, 22010.

12. World Health Organization: WHO briefing on Malaria Treatment Guidelines and artemisinin monotherapies. Geneva: WHO 2006.

13. World Health Organization: Methods for surveillance of antimalarial drug efficacy. Geneva: WHO 2009 [http://whqlibdoc.who.int/publications/2009/ 9789241597531_eng.pdf], ISBN 9789241597531.

14. White NJ: The role of anti-malarial drugs in eliminating malaria. Malar J 2008, 7(Suppl 1):S8.

15. Newton P, van Vugt N, Teja-lsavadharm, Siriyanonda D, Rasameesoroj M, Teerapong P, Ruangveerayuth R, Slight T, Nosten F, Suputtamongkol Y, Looareesuwan S, White NJ: Comparison of oral artesunate and dihydroartemisinin antimalarial bioavailabilities in acute falciparum malaria. Antimicrob Agents Chemother 2002, 46:1125-1127.

16. Rojanawatsirivej C, Vijaykadga S, Amklad I, Wilairatna P, Looareesuwan S: Monitoring the therapeutic efficacy of antimalarials agaist uncomplicated falciparum malaria in Thailand. Southeast Asian J Trop Med Public Health 2003, 34:536-541.

17. Krudsood S, Tangpukdee N, Wilairatana P, Phophak N, Baird JK, Brittenham GM, Looareesuwan S: High-dose primaquine regimens against relapse of Plasmodium vivax malaria. Am J Trop Med Hyg 2008, 78:736-740.

18. Galappaththy GNL, Omari AAA, Tharyan P: Primaquine for preventing relapses in people with Plasmodium vivax malaria (Review). The Cochrane Collaboration. Published by John Wiley \& Sons, Ltd 2008.

19. Krudsood S, Tangpukdee N, Muangchareon S, Thanachartwet V, Luplertlop N, Srivilairit S, Wilairatana P, Kano S, Ringwald, Looareesuwan S: Clinical efficacy of chloroquine versus artemether-lumefantrine for Plasmodium vivax treatment in Thailand. Korean Journal of Parasitology 2007, 45:111-114.
20. World Health Organization: World malaria report 2005. Geneva: WHO 2005, 294.

21. Agyepong IA, Ansah E, Gyapong M, Adjei S, Barnish G, Evans D: Strategies to improve adherence to recommended chloroquine treatment regimes: a quasi-experiment in the context of integrated primary health care delivery in Ghana. Soc Sci Med 2002, 55:2215-2226.

22. World Health Organization: Adherence to long-term therapies: evidence for action. Geneva: WHO 2003, 110.

23. World Health Organization: Monitoring Antimalarial Drug Resistance, Report of a WHO consultation. Geneva, Switzerland 2001.

24. Souares A, Lalou R, Sene I, Sow D, Le Hesran JY: Adherence and effectiveness of drug combination in curative treatment among children suffering uncomplicated malaria in rural Senegal. Trans $R$ Soc Trop Med Hyg 2008, 102:751-758.

25. Vestergaard LS, Ringwald P: Responding to the challenge of antimalarial drug resistance by routine monitoring to update national malaria treatment policies. Am J Trop Med Hyg 2007, 77(Suppl 6):153-159.

26. Microsoft Research: Cell Phone as a Platform for Healthcare Awards. 2008 [http://research.microsoft.com/en-us/um/redmond/about/collaboration/ awards/cellphone-healthcare_awards.aspx].

27. Bureau of Policy and Strategy: Statistics. Nonthaburi: Ministry of Public Health, Thailand 2010 [http://bps.ops.moph.go.th].

28. Bureau of Vector Borne Disease, Department of Disease Control: Vector Borne Disease Reference Laboratory. Nonthaburi: Ministry of Public Health, Thailand 2009.

29. Battistella G: Irregular Migration - World Migration 2008. Rome: International Migration Institute (SIMI) 2008.

30. Linda D, Vasuprasat P: Migrant workers' remittances from Thailand to Cambodia, Lao PDR and Myanmar : synthesis report on survey findings in three countries and good practices. ILO/Japan Project on Managing Cross-border Movement of Labour in Southeast Asia; ILO Regional Office for Asia and the Pacific. - Bangkok: ILO 2010.

31. Congpuonga K, Saipomsudb W, Chompoonuchc C, Niemhomd P, Vinayake S, Satimaia W: Efficacy of a 3-day artesunate-mefloquine combination in the treatment of uncomplicated falciparum malaria in Kanchanaburi province of Thailand. Asian Biomedicine 2010, 4:289-295.

32. Bureau of Vector Borne Disease: Malaria situation. Nonthaburi:Ministry of Public Health of Thailand 2008 [http://www.thaivbd.org/cms].

33. Luxemburger C, Kyaw Ley Thew, White NJ, Webster HK, Kyle DE, Maelankiri L, Chongsuphajaisiddhi T, Nosten F: The epidemiology of malaria in a Karen population on the western border of Thailand. Trans R Soc Trop Med Hyg 1996, 90:105-111.

34. Tipmontree R, Fungladda W, Kaewkungwal J, Tempongko SB, Schelp FP: Migrants and malaria risk factors: A study of the Thai-Myanmar border. Southeast Asian J Trop Med Public Health 2009, 40:1148-1157.

35. Zhou G, Sirichaisinthop J, Sattabongkot J, Jones J, Bjonstad ON, Yan G, Cui $L$ : Spatio-temporal distribution of Plasmodium falciparum amd $P$. vivax malaria in Thailand. Am J Trop Med Hyg 2005, 72:256-262.

36. Patipong S, Yongchaitrakul S: Field efficacy and persistence of Long Lasting Insecticide treated mosquito Nets (LLINs) in comparison with conventional Insecticide Treated mosquito Nets (ITN) against malaria vector in Thailand. J Vector-borne Dis 2008, 5:7-13.

37. Khantikul N, Butraporn P, Kim HS, Leemingsawat S, Tempongko SB, Suwonkerd W: Adherence to antimalarial drug therapy among vivax malaria patients in northern Thailand. J Health Popul Nutr 2009, 27:4-13.

38. White NJ: The management of severe falciparum malaria. Am J Respir Crit Care Med 2003, 167:673-674.

39. Zwang J, Ashley EA, Karema C, D'Alessandro U, Smithuis F, Dorsey G, Janssens B, Mayxay M, Newton P, Singhasivanon P, Stepniewska P, White NJ, Nosten F: Safety and efficacy of dihydroartemisininpiperaquine in falciparum malaria: A prospective multi-centre individual patient data analysis. PLOS ONE 2009, 4:e6358.

40. Hutagalung R, Paiphun L, Ashley EA, McGready R, Brockman A, Thwai KL, Singhasivanon $\mathrm{P}$, Jelinek $\mathrm{T}$, White $\mathrm{NJ}$, Nosten $\mathrm{FH}$ : A randomized trial of artemether-lumefantrine versus mefloquine-artesunate for the treatment of uncomplicated multi-drug resistant Plasmodium falciparum on the western border of Thailand. Malar J 2005, 4:46.

41. Wongsrichanalai C, Sirichaisinthop J, Karwacki JJ, Congpuong K, Miller RS, Pang $L$, Thimasarn K: Drug resistant malaria on the Thai-Myanmar and Thai-Cambodia borders. Southeast Asian J Trop Med Public Health 2001, $32(1)$ 
42. Delacollette C, D'Souza C, Christophel E, Thimasarn K, Abdur R, Bell D,

Dai TC, Gopinath D, Lu S, Mendoza R, Ortega L, Rastogi R, Tantinimitkul C,

Ehrenberg J: Malaria trends and challenges in the greater Mekong subregion. Southeast Asian J Trop Med Public Health 2009, 40(4).

43. The Bill and Melinda Gates Foundation (BMGF) and the World Health

Organization (WHO): Strategy for the containment of artemisinin tolerant malaria parasites in South-East Asia.[http://www.gatesfoundation.org/ Grants-2008/Pages/World-Health-Organization-OPP48821_01.aspx].

44. Booman M, Durrheim DN, La Grange K, Martin C, Mabuza AM, Zitha A, Mbokazi FM, Fraser C, Sharp BL: Using a geographical information system to plan a malaria control programme in South Africa. Bull World Health Organ 2000, 78:1438-1444.

45. Martin C, Curtis B, Fraser C, Sharp B: The use of a GIS-based malaria information system for malaria research and control in South Africa. Health \& Place 2002, 8:227-236.

doi:10.1186/1475-2875-9-237

Cite this article as: Meankaew et al: Application of mobile-technology for disease and treatment monitoring of malaria in the "Better Border Healthcare Programme". Malaria Journal 2010 9:237.

\section{Submit your next manuscript to BioMed Central} and take full advantage of:

- Convenient online submission

- Thorough peer review

- No space constraints or color figure charges

- Immediate publication on acceptance

- Inclusion in PubMed, CAS, Scopus and Google Scholar

- Research which is freely available for redistribution

Submit your manuscript at www.biomedcentral.com/submit 